Research Paper

\title{
New Optimal Needle Entry Angle for Cervical Transforaminal Epidural Steroid Injections: A Retrospective Study
}

\author{
Myong-Hwan Karm¹, Jun Young Park², Doo Hwan Kim², Hyun-Seok Cho², Jae-Young Lee², Koo Kwon², \\ and Jeong Hun Suh2 ${ }^{\bowtie}$ \\ 1. Department of Dental Anesthesiology, Seoul National University Dental Hospital, Seoul, Korea; \\ 2. Department of Anesthesiology and Pain Medicine, Asan Medical Center, University of Ulsan College of Medicine, Seoul, Korea. \\ $\square$ Corresponding author: Jeong Hun Suh, M.D., Ph.D., Department of Anesthesiology and Pain Medicine, Asan Medical Center, University of Ulsan College of \\ Medicine, 88 Olympic-ro 43-gil, Songpa-gu, Seoul 05505, Republic of Korea, Tel: +82-2-3010-5630, Fax: +82-2-470-1363, E-mail: paindrsuh@gmail.com
}

(c) Ivyspring International Publisher. This is an open access article distributed under the terms of the Creative Commons Attribution (CC BY-NC) license (https://creativecommons.org/licenses/by-nc/4.0/). See http://ivyspring.com/terms for full terms and conditions.

Received: 2016.08.05; Accepted: 2016.11.15; Published: 2017.04.08

\begin{abstract}
Objective: A cervical epidural steroid injection is one of the most commonly performed interventions to manage chronic neck pain and cervical radiculopathy. Despite its many severe complications, cervical transforaminal epidural steroid injection (CTFESI) is a clinically necessary modality for managing neck pain and cervical radiculopathy. We aimed in this study to find a safer optimal needle entry angle to decrease the chance of an accidental vertebral artery (VA) puncture even with a proper needle entry angle and to visualize the target of the needle tip.

Methods: This retrospective study included 312 patients with neck pain or cervical radiculopathy who had undergone magnetic resonance imaging scans for diagnosis and treatment. The first line was drawn from the midpoint of the two articular pillars and passed through the exact midline of the spinous process. The second line was drawn parallel to the ventral lamina line (conventional transforaminal approach line, CTAL). The third line was drawn parallel to the ventral margin at the midpoint of the superior articular process's ventral border (new transforaminal approach line, NTAL). The angle of intersection between the midline and CTAL versus with NTAL were measured from both sides (right and left) at C5-6, C6-7, and C7-T1 levels. Also, the distance of CTAL and NTAL from VA were measured from both sides at each level. We examined whether the CTAL and NTAL would penetrate the ipsilateral VA, internal carotid artery (ICA), and internal jugular vein (IJV).

Results: There were significant differences between CTAL and NTAL angles at all levels $(P<0.001)$. There were significant differences between the distance of CTAL and NTAL from VA at all levels $(P<0.001)$. There were also significant differences between the observed frequency of CTAL and NTAL that would penetrate the major ipsilateral vessel (VA, ICA, and IJV) on all levels and sides $(P<0.001 \sim 0.030)$.
\end{abstract}

Conclusion: The angle of NTAL (approximately $70^{\circ}$ ) is safer than the angle of CTAL (approximately $50^{\circ}$ ) when considering vascular injuries to vessels, such as the VA, ICA, and IJV.

Key words: cervical radiculopathy; internal carotid artery; internal jugular vein; needle entry angle; transforaminal epidural steroid injection; vertebral artery.

\section{Introduction}

With a prevalence of $38 \%$ to $48 \%$, neck pain is common in the adult population, and the prevalence of chronic neck pain is $16 \%$ to $22 \%$ [1]. The incidence of cervical radiculopathy is also not rare $[2,3]$. Neck pain and cervical radiculopathy have been shown to be caused by cervical facet joints, the atlanto-axial and atlanto-occipital joints, fascia, ligaments, muscles, nerve roots, and intervertebral discs $[1,4,5]$. There are various modalities of non-invasive therapy for neck pain with or without cervical radiculopathy, such as analgesics, physiotherapy, cervical traction, manual therapy, and exercise therapy [6-8]. However, the effects of these conservative treatments remain uncertain and controversial [6].

Cervical epidural steroid injection (ESI) is one of the most commonly performed interventions to 
manage chronic neck pain and cervical radiculopathy from spinal stenosis, herniated discs, chemical discs, discogenic pain, and chronic pain secondary to post-cervical surgery syndrome $[1,9,10]$. There is moderate evidence in systematic reviews and randomized clinical trials that cervical ESI is effective in reducing neck pain and cervical radiculopathy $[1,9$, 11, 12]. Cervical ESI is performed either by transforaminal or interlaminar approaches [1, 13-15].

More severe complications, such as subdural hematoma, quadriparesis, brainstem herniation, spinal cord injury, brainstem and cervical spinal cord infarction, vertebral artery (VA) perforation, and even death, occur more often with cervical transforaminal epidural steroid injection (CTFESI) than with cervical interlaminar epidural steroid injection (CILESI) [15-26]. Despite these severe complications, CTFESI is a clinically necessary modality for managing neck pain and cervical radiculopathy because it is the target-specific modality requiring the smallest volume to reach the primary site of pathology [4].

Chen et al. asserted that the optimal needle entry angle using the anterior oblique approach for performing CTFESI is approximately $50^{\circ}$ in the supine position [27]. Although that angle allows better visualization of the neural foramen (NF), we were doubtful about the safety of this angle. Actually, the risk of VA perforation remains even when CTFESI is performed using a $50^{\circ}$ angle. We believe that a view with a better visualization of the NF is different from the safest procedure because the target of the needle tip is not the inner side of the NF. CTFESI can be performed if the superior articular process (SAP) is well-exposed because the target of the needle tip is the ventral border of SAP.

Hence, we aimed in this study to identify a safer optimal needle entry angle to decrease chance of an accidental VA puncture and to visualize the target of the needle tip, hypothesizing that the needle entry angle of the SAP's ventral margin is farther away from the VA than the angle of the lamina's ventral margin.

\section{Methods}

This retrospective observational study was conducted in the pain management clinic and was approved by the institutional review board of Asan Medical Center (approval number, 2016-0230). The necessity for obtaining informed consent was waived because the investigators only retrospectively reviewed the electronic medical record data in this study. The cases in this retrospective study included 312 patients with neck pain or cervical radiculopathy in 2015 who had undergone magnetic resonance imaging (MRI) scans for diagnosis and treatment.
Inclusion criteria included the following: (1) patient age $\geq 20$ years; (2) had visited the Asan Medical Center for treatment of neck pain or cervical radiculopathy; (3) available results of cervical MRI scans; and (4) a diagnosed cervical radiculopathy, HIVD, or spinal stenosis. Exclusion criteria included (1) patient age $<20$ years; (2) history of prior cervical spine surgery; and (3) having another anatomical abnormality on cervical MRI scans.

Data were collected by measuring the angles from the axial T2-weighted imaging from C5-6, C6-7, and C7-T1. The axial section image was selected that best observed the inner border of both the foramen and laminar among the intervertebral disc level images at each level. The angles on the axial sections of the MRI imaging were measured using Picture Archiving and Communication System (PACS) software. The first line was drawn from the midpoint of the two articular pillars and passed through the exact midline of the spinous process. In the case of a bifid spinous process, the line was drawn through the midpoint of the bifid process. The second line was drawn parallel to the ventral lamina line (conventional transforaminal approach line, CTAL) [27]. The third line was drawn parallel to the ventral margin at the midpoint of the SAP's ventral border (new transforaminal approach line, NTAL). The angle of intersection between the midline and CTAL, as well as that between the midline and NTAL were measured by PACS software from both sides (right and left) at C5-6, C6-7, and C7-T1 levels. Also, the distance of CTAL and NTAL from VA were measured from both sides at each level. We examined whether the CTAL and NTAL penetrated the ipsilateral VA, internal carotid artery (ICA), and internal jugular vein (IJV; Fig. 1).

\section{Data Analysis and Statistical Methods}

We divided the study subjects into two groups, CTAL and NTAL. To assess angle and distances differences between the two groups, angles and distances were compared using paired t-tests. Categorical variables were compared using the $\chi^{2}$ test or Fisher's exact test to assess the difference between the frequencies that the two lines penetrated the major vessels, as appropriate. Continuous variables are presented as means with standard deviation (SD), 95\% confidence intervals (CI), or medians with the interquartile range (IQR). Categorical variables are presented as absolute numbers and percentages. Analyses were performed using SPSS Statistics version 21 (IBM Corp., Armonk, NY). A two-tailed P-value $<0.05$ was considered to indicate a statistically significant difference. 


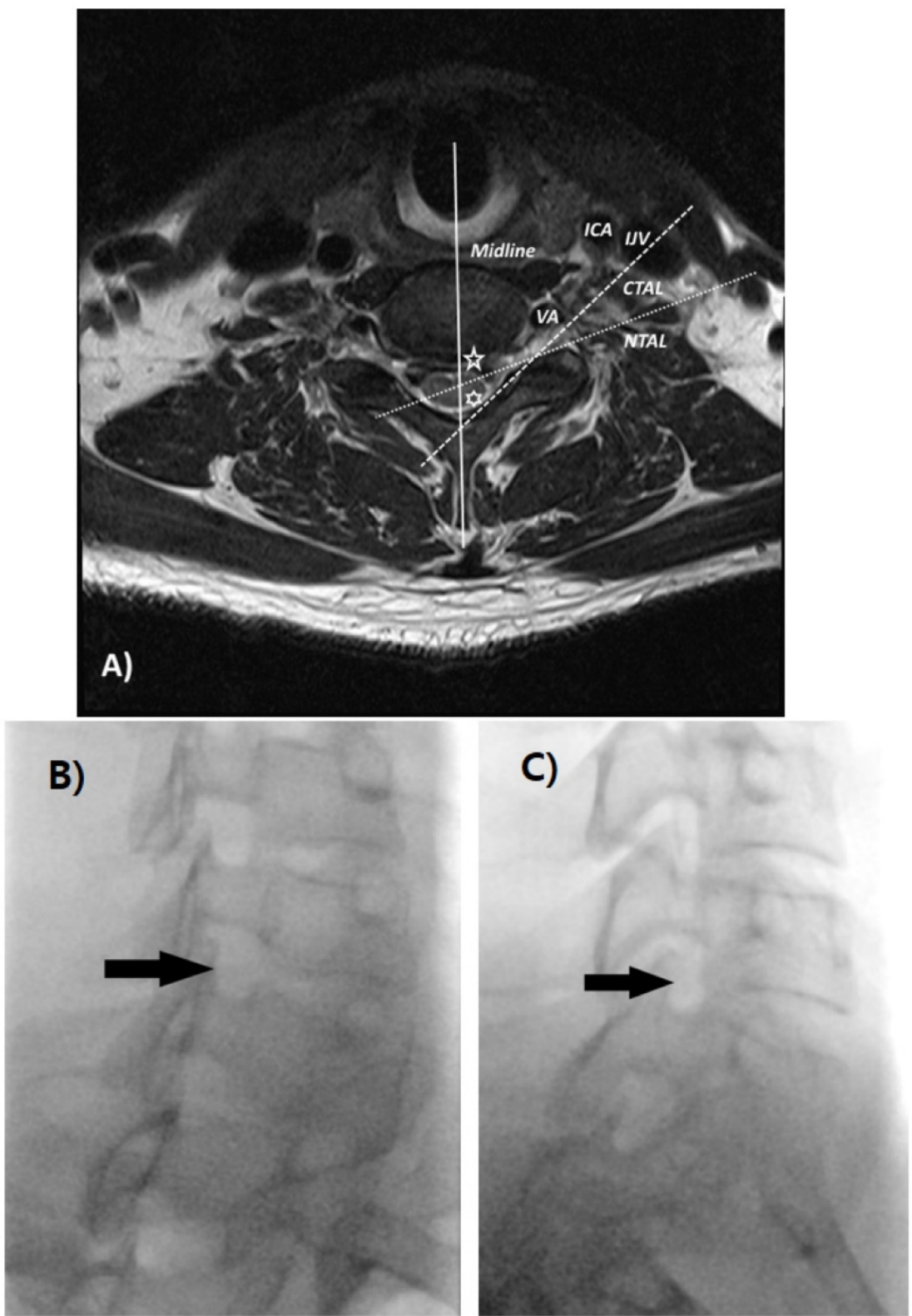

Figure 1. A) The midline (solid line, blue) was drawn from the midpoint of the two articular pillars and to pass through the midline of the spinous process. The CTAL (dashed line, yellow) was drawn parallel to the ventral lamina line, and the NTAL (dotted line, white) was drawn parallel to the ventral margin of the superior articular process (SAP). A five-pointed star (green) represents the angle between the midline and NTAL, and the six-pointed star (green) represents the angle between the midline and CTAL. Different fluoroscopic views in a single subject that were taken as part of study; B) oblique $50^{\circ} \mathrm{C}$ ) oblique $70^{\circ}$. CTAL = conventional transforaminal approach line; NTAL = new transforaminal approach line; VA = vertebral artery; ICA = internal carotid artery; IJV = internal jugular vein.

\section{Results}

We screened patients who had undergone MRI scans during 2015 for neck pain or radiculopathy in their upper extremities at Asan Medical Center. A total of 312 patients who met the inclusion criteria were retrospectively reviewed. Patient demographic data are presented in Table 1 . There were 162 male and 150 female subjects with a mean age (IQR) of 50 (44-55) years. The primary diagnosis among these patients was cervical radiculopathy $(198,63.5 \%)$, a herniated intervertebral disc (HIVD; 79, 25.3\%), or Table 1. Demographic data

\begin{tabular}{ll}
\hline Parameters & $\mathrm{N}=312$ \\
\hline Age (years) & $50.0(44.0-55.0)$ \\
Gender (male / female) & $162(51.9 \%) / 150(48.1 \%)$ \\
Diagnosis & $198(63.5 \%)$ \\
Cervical radiculopathy & $79(25.3 \%)$ \\
Cervical HIVD & $35(11.2 \%)$ \\
Cervical spinal stenosis &
\end{tabular}

Data are expressed medians (interquartile range) or numbers (\%).

HIVD $=$ Herniated intervertebral disc spinal stenosis $(35,11.2 \%)$. 
The angle between the two lines and differences in these two angles are listed in Table 2. The CTAL angles on the right C5-6, C6-7, C7-T1, left C5-6, C6-7, and C7-T1 were the following: $50.0 \pm 5.6,50.2 \pm 4.9$, $50.9 \pm 5.0,51.1 \pm 5.5,50.3 \pm 5.0$, and $50.9 \pm 5.5$, respectively. The NTAL angles on the right C5-6, C 6-7, C7-T1, left C5-6, C6-7, and C7-T1 were as follows: $66.2 \pm 7.2,71.4 \pm 6.6,73.1 \pm 6.9,66.0 \pm 7.5,70.8 \pm 6.0$, and $72.0 \pm 7.4$, respectively. There were significant differences between the CTAL and NTAL angles at all levels $(\mathrm{P}<0.001)$. The estimated difference $(95 \% \mathrm{CI})$ on the right C5-6, C6-7, C7-T1, left C5-6, C6-7, and C7-T1 were 16.2 (15.2-17.2), 21.2 (20.3-22.1), 22.2 (21.2-23.2), 14.9 (14.0-15.9), 20.5 (19.7-21.4), and 21.1 (20.1-22.2), respectively.

Table 2. Angle of the conventional transforaminal approach line (CTAL) and the new transforaminal approach line (NTAL) at each level

\begin{tabular}{llllll}
\hline Level & Side & $\begin{array}{l}\text { CTAL } \\
\text { angle }\end{array}$ & NTAL angle & $\begin{array}{l}\text { Estimated difference } \\
(95 \% \mathrm{CI})\end{array}$ & P-value \\
\hline C5-6 & Right & $50.0 \pm 5.6$ & $66.2 \pm 7.2$ & $16.2(15.2-17.2)$ & $<.001$ \\
& Left & $51.1 \pm 5.5$ & $66.0 \pm 7.5$ & $14.9(14.0-15.9)$ & $<.001$ \\
C6-7 & Right & $50.2 \pm 4.9$ & $71.4 \pm 6.6$ & $21.2(20.3-22.1)$ & $<.001$ \\
& Left & $50.3 \pm 5.0$ & $70.8 \pm 6.0$ & $20.5(19.7-21.4)$ & $<.001$ \\
C7-T1 & Right & $50.9 \pm 5.0$ & $73.1 \pm 6.9$ & $22.2(21.2-23.2)$ & $<.001$ \\
& Left & $50.9 \pm 5.5$ & $72.0 \pm 7.4$ & $21.1(20.1-22.2)$ & $<.001$ \\
\hline \multicolumn{7}{l}{ Data are expressed as the mean \pm standard deviation. CTAL = conventional } \\
transforaminal approach line, NTAL = new transforaminal approach line
\end{tabular}

The distance of CTAL and NTAL from VA are listed in Table 3. The distance ( $\mathrm{mm}$ ) of CTAL from VA on the right C5-6, C6-7, C7-T1, left C5-6, C6-7, and C7-T1 were the following: $3.3 \pm 1.8,3.4 \pm 2.0,4.8 \pm 2.9$, $3.1 \pm 1.8,3.2 \pm 2.1$, and $5.3 \pm 3.5$, respectively. The distance $(\mathrm{mm})$ of NTAL from VA on the right C5-6, C6-7, C7-T1, left C5-6, C6-7, and C7-T1 were the following: $4.2 \pm 1.6,6.2 \pm 2.1,11.0 \pm 3.3,4.2 \pm 1.4,5.8 \pm$ 2.2 , and $11.0 \pm 3.5$, respectively. There were significant differences between the distance of CTAL and NTAL from VA at all levels $(\mathrm{P}<0.001)$. The estimated difference (95\% CI) on the right C5-6, C6-7, C7-T1, left C5-6, C6-7, and C7-T1 were 1.1 (0.8-1.3), 2.9 (2.7-3.2),
6.1 (5.7-6.4), $1.0 \quad(0.8-1.2), 2.7 \quad(2.4-3.0)$, and 5.8 (5.5-6.2), respectively.

Table 3. Distance of the conventional transforaminal approach line (CTAL) and the new transforaminal approach line (NTAL) from vertebral artery (VA) at each level

\begin{tabular}{|c|c|c|c|c|c|}
\hline Level & Side & $\begin{array}{l}\text { CTAL - VA } \\
(\mathrm{mm})\end{array}$ & $\begin{array}{l}\text { NTAL - VA } \\
(\mathrm{mm})\end{array}$ & $\begin{array}{l}\text { Estimated difference } \\
(95 \% \mathrm{CI})\end{array}$ & P-value \\
\hline \multirow[t]{2}{*}{ C5-6 } & Right & $3.3 \pm 1.8$ & $4.2 \pm 1.6$ & $1.1(0.8-1.3)$ & $<.001$ \\
\hline & Left & $3.1 \pm 1.8$ & $4.2 \pm 1.4$ & $1.0(0.8-1.2)$ & $<.001$ \\
\hline \multirow[t]{2}{*}{ C6-7 } & Right & $3.4 \pm 2.0$ & $6.2 \pm 2.1$ & $2.9(2.7-3.2)$ & $<.001$ \\
\hline & Left & $3.2 \pm 2.1$ & $5.8 \pm 2.2$ & $2.7(2.4-3.0)$ & $<.001$ \\
\hline \multirow[t]{2}{*}{ C7-T1 } & Right & $4.8 \pm 2.9$ & $11.0 \pm 3.3$ & $6.1(5.7-6.4)$ & $<.001$ \\
\hline & Left & $5.3 \pm 3.5$ & $11.0 \pm 3.5$ & $5.8(5.5-6.2)$ & $<.001$ \\
\hline
\end{tabular}

Data are expressed as the mean \pm standard deviation. CTAL $=$ conventional transforaminal approach line, NTAL = new transforaminal approach line, $\mathrm{VA}=$ vertebral artery

The observed frequencies that the line penetrated a major ipsilateral vessel (VA, ICA, and IJV) are presented in Table 4 . The frequencies that CTAL penetrated the ipsilateral VA on the right C5-6, C6-7, C7-T1, left C5-6, C6-7, and C7-T1 were the following: $11(3.5 \%), 17(5.4 \%), 14(4.5 \%), 8(2.6 \%), 24$ $(7.7 \%)$, and $25(8 \%)$, respectively. The frequencies that NTAL penetrated the ipsilateral VA at all levels and sides were zero. The frequencies that CTAL penetrated the ipsilateral ICA on the right C5-6, C6-7, C7-T1, left C5-6, C6-7, and C7-T1 were the following: $60(19.2 \%), 26(8.3 \%), 7$ (2.2\%), 45 (14.4\%), 34 (10.9\%), and $6(1.9 \%)$, respectively. The frequencies on the right and left C5-6 that NTAL penetrated the ipsilateral ICA were $4(1.3 \%)$ and $5(1.6 \%)$, respectively. The frequency at all other levels and sides was zero. The frequencies that CTAL penetrated the ipsilateral IJV on the right C5-6, C6-7, C7-T1, left C5-6, C6-7, and C7-T1 were the following: 204 (65.4\%), $176(56.4 \%), 71(22.8 \%), 188(60.3 \%), 142(45.5 \%)$, and $52(16.7 \%)$, respectively. The frequencies of NTAL C7-T1 were $125(40.0 \%), 36(11.5 \%), 5(1.6 \%), 90$ (28.8\%), 24 (7.7\%), and $2(0.6 \%)$, respectively. There were significant differences between the observed frequency that CTAL versus NTAL penetrated the major ipsilateral vessel (VA, ICA, and IJV) on all levels and sides $(\mathrm{P}<0.001 \sim 0.030)$.

Table 4. Observed frequency of the lines penetrating the major ipsilateral vessel

\begin{tabular}{|c|c|c|c|c|c|c|c|c|c|c|}
\hline \multirow[t]{2}{*}{ Level } & \multirow[t]{2}{*}{ Side } & \multicolumn{2}{|l|}{ VA } & \multirow[t]{2}{*}{ P-value } & \multicolumn{2}{|l|}{ ICA } & \multirow[t]{2}{*}{ P-value } & \multicolumn{2}{|l|}{ IJV } & \multirow[t]{2}{*}{ P-value } \\
\hline & & CTAL & NTAL & & CTAL & NTAL & & CTAL & NTAL & \\
\hline \multirow[t]{2}{*}{ C5-6 } & Right & $11(3.5 \%)$ & $0(0 \%)$ & $<.001$ & $60(19.2 \%)$ & $4(1.3 \%)$ & $<.001$ & $204(65.4 \%)$ & $125(40.0 \%)$ & $<.001$ \\
\hline & Left & $8(2.6 \%)$ & $0(0 \%)$ & $<.001$ & 45 (14.4\%) & $5(1.6 \%)$ & $<.001$ & $188(60.3 \%)$ & $90(28.8 \%)$ & $<.001$ \\
\hline \multirow[t]{2}{*}{ C6-7 } & Right & $17(5.4 \%)$ & $0(0 \%)$ & $<.001$ & $26(8.3 \%)$ & $0(0 \%)$ & $<.001$ & $176(56.4 \%)$ & $36(11.5 \%)$ & $<.001$ \\
\hline & Left & $24(7.7 \%)$ & $0(0 \%)$ & $<.001$ & $34(10.9 \%)$ & $0(0 \%)$ & $<.001$ & $142(45.5 \%)$ & $24(7.7 \%)$ & $<.001$ \\
\hline \multirow[t]{2}{*}{ C7-T1 } & Right & $14(4.5 \%)$ & $0(0 \%)$ & $<.001$ & $7(2.2 \%)$ & $0(0 \%)$ & .015 & $71(22.8 \%)$ & $5(1.6 \%)$ & $<.001$ \\
\hline & Left & $25(8 \%)$ & $0(0 \%)$ & $<.001$ & $6(1.9 \%)$ & $0(0 \%)$ & .030 & $52(16.7 \%)$ & $2(0.6 \%)$ & $<.001$ \\
\hline
\end{tabular}

Data are expressed as numbers (\%). CTAL $=$ conventional transforaminal approach line; ICA $=$ internal carotid artery; IJV $=$ internal jugular vein; NTAL $=$ new

transforaminal approach line; $\mathrm{VA}=$ vertebral artery 


\section{Discussion}

Finding safer methods of performing the CTFESI procedure has required constant efforts. The proper needle entry angle for CTFESI was proposed by Chen et al. [27]. Although their recommendation represents the angle that allows optimal visualization of the NF, this angle may not be optimal for CTFESI. The risk of VA perforation always exists due to variable anomalous locations of the VA [28-30]. Before performing CTFESI, evaluating for possible variations of cervical vascular anatomy through an MRI or CT would be helpful to minimize the risk of adverse events.

In our current study, we measured and evaluated the needle entry angle of two methods (CTAL and NTAL) at three levels (C5-6, C6-7, and C7-T1) on both sides (right and left) of 312 patients. The needle entry angle of CTAL was approximately $50^{\circ}$, while that of NTAL was approximately $70^{\circ}$. We believe that this difference of angle occurred because we have chosen the better visualization view of the SAP not of the NF. A slight difference in the needle entry angle at the skin could increase the chance of a VA puncture. We demonstrated that the needle position when the NTAL method is used is farther away from the VA than when the CTAL method is performed (Table 3). Because the complication of an incidental VA puncture is fatal, we are obligated to seek the safer method. We consider the NTAL method $\left(70^{\circ}\right.$, approximately) to be safer because of its lower expected risk of an accidental VA puncture.

We also analyzed the frequency of the angle penetrating the ipsilateral VA, ICA, and IJV, and found that the incidence of the angle of CTAL penetrating the ipsilateral VA, ICA, and IJV was greater than that of NTAL on all levels and sides. It is impressive that the frequencies that NTAL penetrated the ipsilateral VA at all levels and sides were zero. There was a significant difference in the incidence of the angle penetrating the ipsilateral VA, ICA, and IJV $(\mathrm{P}<0.001 \sim 0.030)$. These complications involving VA, ICA or IJV injury are also major problems and have to be prevented to decrease the prevalence of VA, ICA or IJV puncture. We recommend that an MRI or CT should be evaluated for a vascular anatomical abnormality, such as VA, ICA, and IJV, before performing CTFESI if possible. This action could decrease the chance of vascular injury complications. Also, we thought that measuring the expected needle length from the VA and the needle entry angle on the MRI image would be helpful to develop a safer procedure.

Our study had several limitations of note. First, this study was designed to be retrospective and observational and was not controlled or blinded. Second, we measured and analyzed the needle entry angle, but we cannot yet acquire real practice data using our new angle. Although we have performed CTFESI using the new angle at our center, additional time will be required to collect data using this new angle. We are planning to perform a randomized controlled trial to evaluate the optimal entry needle angle.

\section{Conclusion}

The angle of NTAL (approximately $70^{\circ}$ ) is safer than the angle of CTAL (approximately $50^{\circ}$ ) when considering vascular injuries to vessels, such as the VA, ICA, and IJV. However, more research will be needed to develop a consensus on the safe needle entry angle because of a relative lack of research to date on the optimal needle entry angle for CTFESI.

\section{Abbreviations}

ESI: epidural steroid injection; VA: vertebral artery; CTFESI: cervical transforaminal epidural steroid injection; CILESI: cervical interlaminar epidural steroid injection; NF: neural foramen; SAP: superior articular process; MRI: magnetic resonance imaging; PACS: Picture Archiving and Communication System; CTAL: conventional transforaminal approach line; NTAL: new transforaminal approach line; ICA: internal carotid artery; IJV: internal jugular vein; SD: standard deviation; CI: confidence intervals; IQR: interquartile range; HIVD: herniated intervertebral disc.

\section{Acknowledgments}

We are grateful to the pain clinic members of Asan medical center for the collection of data.

\section{Competing Interests}

The authors have declared that no competing interest exists.

\section{References}

1. Diwan S, Manchikanti L, Benyamin RM, et al. Effectiveness of cervical epidural injections in the management of chronic neck and upper extremity pain. Pain Physician. 2012; 15: E405-34.

2. Radhakrishnan K, Litchy WJ, O'Fallon WM, et al. Epidemiology of cervical radiculopathy. A population-based study from Rochester, Minnesota, 1976 through 1990. Brain. 1994; 117 ( Pt 2): 325-35.

3. Faught RW, Church EW, Halpern $\mathrm{CH}$, et al. Long-term quality of life after posterior cervical foraminotomy for radiculopathy. Clin Neurol Neurosurg. 2016; 142: 22-5.

4. Manchikanti L, Nampiaparampil DE, Candido KD, et al. Do cervical epidural injections provide long-term relief in neck and upper extremity pain? A systematic review. Pain Physician. 2015; 18: 39-60.

5. Kuijper B. Cervical radiculopathy. Ned Tijdschr Tandheelkd. 2014; 121: 483-6.

6. Salt E, Wright C, Kelly S, et al. A systematic literature review on the effectiveness of non-invasive therapy for cervicobrachial pain. Man Ther. 2011; 16: 53-65.

7. Zarghooni K, Beyer F, Siewe J, et al. The orthotic treatment of acute and chronic disease of the cervical and lumbar spine. Dtsch Arztebl Int. 2013; 110: 737-42. 
8. Thoomes EJ, Scholten-Peeters W, Koes B, et al. The effectiveness of conservative treatment for patients with cervical radiculopathy: a systematic review. Clin J Pain. 2013; 29: 1073-86.

9. Benyamin RM, Singh V, Parr AT, et al. Systematic review of the effectiveness of cervical epidurals in the management of chronic neck pain. Pain Physician. 2009; 12: 137-57.

10. Castagnera L, Maurette P, Pointillart V, et al. Long-term results of cervical epidural steroid injection with and without morphine in chronic cervical radicular pain. Pain. 1994; 58: 239-43.

11. Manchikanti L, Cash KA, Pampati V, et al. A randomized, double-blind, active control trial of fluoroscopic cervical interlaminar epidural injections in chronic pain of cervical disc herniation: results of a 2-year follow-up. Pain Physician. 2013; 16: 465-78.

12. Stav A, Ovadia L, Sternberg A, et al. Cervical epidural steroid injection for cervicobrachialgia. Acta Anaesthesiol Scand. 1993; 37: 562-6.

13. Manchikanti L, Abdi S, Atluri S, et al. An update of comprehensive evidence-based guidelines for interventional techniques in chronic spinal pain. Part II: guidance and recommendations. Pain Physician. 2013; 16: S49-283.

14. Manchikanti L, Falco FJ, Diwan S, et al. Cervical radicular pain: the role of interlaminar and transforaminal epidural injections. Curr Pain Headache Rep. 2014; $18: 389$.

15. Landa J, Kim Y. Outcomes of interlaminar and transforminal spinal injections. Bull NYU Hosp Jt Dis. 2012; 70: 6-10.

16. Scanlon GC, Moeller-Bertram T, Romanowsky SM, et al. Cervical transforaminal epidural steroid injections: more dangerous than we think? Spine. 2007; 32: 1249-56.

17. Rozin L, Rozin R, Koehler SA, et al. Death during transforaminal epidural steroid nerve root block (C7) due to perforation of the left vertebral artery. Am J Forensic Med Pathol. 2003; 24: 351-5.

18. Ruppen W, Hugli R, Reuss S, et al. Neurological symptoms after cervical transforaminal injection with steroids in a patient with hypoplasia of the vertebral artery. Acta Anaesthesiol Scand. 2008; 52: 165-6.

19. Lee JH, Lee JK, Seo BR, et al. Spinal cord injury produced by direct damage during cervical transforaminal epidural injection. Reg Anesth Pain Med. 2008; 33: 377-9.

20. Muro K, O'Shaughnessy B, Ganju A. Infarction of the cervical spinal cord following multilevel transforaminal epidural steroid injection: case report and review of the literature. J Spinal Cord Med. 2007; 30: 385-8.

21. Tiso RL, Cutler T, Catania JA, et al. Adverse central nervous system sequelae after selective transforaminal block: the role of corticosteroids. Spine J. 2004; 4: $468-74$.

22. Karasek M, Bogduk N. Temporary neurologic deficit after cervical transforaminal injection of local anesthetic. Pain Med. 2004; 5: 202-5.

23. Rathmell JP, Aprill C, Bogduk N. Cervical transforaminal injection of steroids. Anesthesiology. 2004; 100: 1595-600

24. Huston CW. Cervical epidural steroid injections in the management of cervical radiculitis: interlaminar versus transforaminal. A review. Curr Rev Musculoskelet Med. 2009; 2: 30-42.

25. Sadacharam K, Petersohn JD, Green MS. Inadvertent Subdural Injection during Cervical Transforaminal Epidural Steroid Injection. Case Rep Anesthesiol. 2013; 2013: 847085.

26. Baker R, Dreyfuss $P$, Mercer $S$, et al. Cervical transforaminal injection of corticosteroids into a radicular artery: a possible mechanism for spinal cord injury. Pain. 2003; 103: 211-5.

27. Chen B, Rispoli L, Stitik TP, et al. Optimal needle entry angle for cervical transforaminal epidural injections. Pain Physician. 2014; 17: 139-44.

28. Lee JK, Chae KW, Ju CI, et al. Acute Cervical Subdural Hematoma with Quadriparesis after Cervical Transforaminal Epidural Block. J Korean Neurosurg Soc. 2015; 58: 483-6.

29. Jung H, Lim JA, Park KB, et al. Computed tomography-guided cervical selective transforaminal epidural block for a patient with bilateral anatomical variations of vertebral artery -a case report. Korean J Anesthesiol. 2013; 65: $468-72$.

30. Shin HY, Park JK, Park SK, et al. Variations in Entrance of Vertebral Artery in Korean Cervical Spine: MDCT-based Analysis. Korean J Pain. 2014; 27: 266-70. 